\title{
«A MI MORADA OSCURA, DESMANTELADA Y FRÍA». POSLUGARES, ESTADO Y LITERATURA: LA CASA DE ROSALÍA CASTRO ${ }^{1}$
}

\author{
«TO MY BLEAK, SHABBY, AND COLD ABODE». POST-PLACE, STATE AND \\ LITERATURE: THE HOUSE OF ROSALÍA CASTRO
}

Fernando CABO ASEGUINOLAZA

Universidade de Santiago de Compostela

Resumen: La casa de A Matanza en Padrón (A Coruña), el lugar de fallecimiento de Rosalía Castro en 1885 y sede actual de la Fundación que lleva su nombre, es la principal referencia para una consideración de lo que llamamos poslugar. Los poslugares literarios se analizan desde su condición de figuras de la memoria pública, esto es, como espacios conmemorativos definidos por su condición de escenarios de acontecimientos ficticios o biográficos de relevancia literaria. Estas formas de escenificación de una topografía literaria se muestran reveladoras de conexiones relevantes entre literatura y Estado. En este caso, la atención se dirigirá en especial al vínculo problemático entre el lugar de A Matanza y el último libro de Rosalía Castro, En las orillas del Sar (1884).

Palabras clave: geografía literaria; poslugar; casas de escritores; Rosalía Castro; En las orillas del Sar.

Abstract: The aim of this paper is to reflect upon what we propose to term as literary 'postplace'. A representative case is laid out: that of the house in A Matanza (Padrón, Spain), where Rosalía Castro died in 1885, and which has been serving as a museum and Foundation dedicated to the author's life since 1972. Literary post-places are here defined as figures of public memory -commemorative sites that served as a backdrop for fictional or biographical events (very frequently related with an author's life) that have had important literary implications. In this light, post-place can be quite revealing of the connections linking the State and the institutionalisation of literary works and authors. To elucidate these processes, the controversial relationship between A Matanza and Castro's last book Beside the River Sar (En las orillas del Sar, 1884) will be analysed.

Keywords: literary geography; post-place; writer's houses; Rosalía Castro; En las orillas del Sar.

\footnotetext{
${ }^{1}$ Este trabajo se vincula al proyecto de investigación "Cartografías del afecto y usos públicos de la memoria: un análisis geoespacial de la obra de Rosalía Castro” (FFI2017-82742-P), financiado por el MINECO (Gobierno de España).
} 
$\mathrm{E}$ 1 propósito inicial de este artículo es profundizar en la formulación del concepto de poslugar, incidiendo para ello en algunas de sus conexiones con el Estado, uno de los marcos determinantes de la noción misma de literatura. Los que denominaremos poslugares son a menudo espacios públicos de memoria o de conmemoración, dispuestos para ser vistos o visitados por su asociación con determinados acontecimientos o experiencias previas que en ellos han tenido lugar, muchas veces en el ámbito de la ficción. Es así con las casas de muchos escritores, convertidas en museos o sedes de instituciones culturales, o con determinados espacios que se identifican y señalan como escenarios literarios. Son lugares, pero teatralizados, próximos al simulacro y orientados hacia formas diversas de consumo, y por lo general se asocian a contextos institucionales más amplios en los que cobran su sentido. Tienen que ver con una zona de transición entre espacios privados o de intimidad y espacios públicos, regidos por lógicas de representación no siempre compatibles. Se sitúan a menudo también en un territorio limítrofe entre los espacios ficcionalizados, de orden simbólico, y los concretos y materiales. El tratamiento de la relación entre la literatura y el Estado desde la consideración de los poslugares, no puede ser aquí más que parcial y limitado, aunque la forma en que estos ponen en evidencia la dimensión cultural e ideológica de determinados entornos resulta muy propicia para abrir vías de reflexión interesantes.

\section{Hacia una caracterización de los poslugares literarios}

Los poslugares son de naturaleza muy distinta: históricos, religiosos, cinematográficos, patrimoniales, políticos... No obstante, los emplazamientos que aquí interesan son en lo fundamental lugares literarios, los cuales poseen, a su vez, una condición un tanto difusa en la medida en que suelen incluir tanto lugares representados, asociados a la geografía o topografía interna de los textos, como lugares biográficos, definidos así a partir de su vinculación con distintos episodios vitales de los autores. Esta situación, entre lo biográfico y lo textual, de los lugares literarios, los hace especialmente propicios para la constitución efectiva de poslugares, ya que no es infrecuente que la conexión entre los espacios ficcionales y los sociales asociados con el mundo real descanse sobre un elemento biográfico. Fueron, ahí radica la posibilidad de su funcionamiento como poslugares, bien escenarios de escritura, bien escenarios representados en la escritura; y, en ocasiones, ambas cosas al tiempo. Una cuestión especialmente relevante para el análisis de los poslugares literarios es, de hecho, la relación tensa entre la topografía interna y externa de los textos; y, más en particular, la relación entre la representación interna o ficticia de los lugares y la constitución de lugares públicos a partir de ellos. Podría uno preguntarse, por ejemplo, si las topografías internas, y sus lógicas de representación ficcional son, por así decir, resistentes a las topografías públicas; o si, por el contrario, constituyen un primer paso en una lógica de abstracción que conduce a la constitución del poslugar. 
Una formulación inicial de la noción de poslugar fue anticipada en un trabajo anterior en torno a la novela Memoria da choiva, de Pedro Feijoo (2013): un thriller literario, cuya acción se sitúa en el mismo año de su escritura. Los espacios articulados alrededor de la figura y obra de Rosalía Castro la topografía rosaliana - resultaban en esta novela un sustento fundamental de la trama. A ese propósito, me refería a los procesos simbólicos que invisten los antiguos lugares con una nueva dimensión institucional e implican una alteración profunda - física, y sobre todo relacional e ideológica - de los espacios originarios (Cabo Aseguinolaza, en prensa). Los poslugares, en este sentido, resultan de un ejercicio intenso de postproducción. Rosalía Castro será ahora también un punto de referencia en esta reflexión, y, de forma particular, el poslugar rosaliano por excelencia: la conocida como casa de A Matanza, en la aldea con ese nombre de la parroquia padronesa de Iria Flavia, donde la autora murió el 15 de julio de 1885 tras haberla habitado, a medias retirada, los últimos años de su vida y que es hoy en día Casa-Museo y sede de la Fundación Rosalía Castro ${ }^{2}$. Fue también un lugar de escritura, donde con toda probabilidad produjo una buena parte de los poemas de En las orillas del Sar (1884).

Cuando hablamos de poslugares literarios, estamos tratando de la inscripción de la ficción en el mundo a través de la acotación y señalamiento de enclaves simbólicos con una referencialidad literaria definida. De forma coincidente o no con lo recién indicado, pueden consistir también en la fijación de enclaves, no menos simbólicos en su configuración, que trasladan una referencia biográfica por su condición atribuida de entorno vital o de experiencia de un artista o autor literario. Estamos, sin duda, ante una de las formas de visibilización y modelación cultural más llamativas, especialmente por lo que implica de socialización institucionalizada de un determinado corpus literario y de canonización material y explícita de algunas de sus instancias. Una hipótesis inicial podría apuntar, de hecho, al relevante papel de los poslugares literarios en la interrelación funcional entre literatura y Estado en la época contemporánea.

Aunque con antecedentes antiguos ${ }^{3}$, el fenómeno adquirió, de hecho, una extensión y características del todo particulares en un momento mucho más cercano. Fue desarrollándose de manera progresiva a partir de la segunda mitad del siglo XVIII, en un proceso bastante preciso que se ha estudiado sobre todo para el caso británico. Se encuentra asociado de manera muy estrecha a los primeros tanteos de la geografía literaria en el siglo XIX y, lo que es casi decir lo mismo, a los programas de turismo cultural de primer momento, asociados a las hoy en día omnipresentes y variopintas rutas literarias, pero también a otros medios como guías, ensayos o mapas. En suma, los poslugares literarios adquieren su marchamo característico a través de su interrelación con un intenso y por veces exuberante «clutter of interrelated modes», en palabras de Nicola J. Watson (2009: 3). De acuerdo con esta investigadora, la expansión de tales conexiones entre literatura y territorio, paralela a la configuración de la moderna institución literaria, habría constituido una fase previa al desarrollo de otros «systems of memorialisation», entre ellos los de carácter «official and topographical» en

\footnotetext{
${ }^{2}$ Consúltese la web oficial de la Fundación e Casa-Museo Rosalía de Castro: http://rosalia.gal/.

${ }^{3}$ Como, por ejemplo, los relacionados con la veneración laica en torno a la figura del Petrarca del Canzoniere en el entorno de Aviñón desde los primeros decenios del siglo XVI. Para este y otros ejemplos, véanse los estudios reunidos por Harald Hendrix (2012).
} 
forma de monumentos, placas, nombres de calles y otros espacios significativos, como la erección de panteones de personalidades ilustres.

Estos procedimientos poseen una función de marcación político-identitaria del territorio, que queda así no solo ligado a una selección canónica de autores, sino teñido de forma indirecta por las connotaciones derivadas de la interpretación del territorio que deriva de la escritura de este elenco cultural. No obstante, hay que tener en cuenta también la conversión de muchos de estos emplazamientos en objeto de consumo simbólico y también directamente económico en el sentido más estricto del término. No hay una contradicción entre todo ello, al menos en lo que implica de socialización del capital literario y de su inversión productiva. Uno de los rasgos más reseñables de los poslugares es, precisamente, su ductilidad para funcionar en distintos niveles y con propósitos aparentemente muy alejados entre sí y de apariencia por veces contradictoria. En este sentido, resulta llamativo el hecho de que estas formas de interrelación entre literatura y lugares no haya decrecido en absoluto, más bien lo contrario. Pensando en el caso concreto de las casas de escritores, señala uno de los mejores conocedores de fenómeno: «Though recognized and revered as memorial sites over many centuries, in recent years writer's houses have grown into a major asset of heritage tourism» (Hendrix, 2012: 1), con la consecuencia de su acelerada proliferación internacional, sobre todo a partir de los años setenta del pasado siglo.

Los lugares literarios subrayan el valor distintivo de cada uno de los entornos asociados y, por tanto, su atractivo como objeto de consumo para un cierto sector de la industria turística. Aunque, al menos en un contexto capitalista, no exista una incompatibilidad evidente entre los procesos de estatalización y mercantilización, la función memorialística de estos emplazamientos parece distinta en cada caso. Agnes Heller recordaba que el arte asumió un papel muy relevante en el abastecimiento de memoria cultural para el Estado a partir del siglo XVIII, fundamentalmente a través de la sociedad civil y de la adopción de formas tomadas muchas veces de las prácticas religiosas; entre ellas, las peregrinaciones a los lugares consagrados a la memoria de los principales referentes de la cultura nacional, como la casa de Goethe en Weimar. Pero también apuntaba Heller que «this kind of cult has assumed a cosmopolitan character as the places of remembrance became also touristic attractions» (Heller, 2001: 142), lo cual, en la medida en los inscribiría en el ámbito del mercado, volcado sobre el interés y el futuro-presente, los enajenaría de su función como figuras de la memoria cultural.

Puede ser así, o no. En cualquier caso, la tensión entre el funcionamiento como bien de consumo cultural o turístico y su relevancia como lugares de memoria es una cuestión de primer orden, que, más que a su incompatibilidad radical, apunta a la capacidad de los poslugares para funcionar simultáneamente en contextos en apariencia diversos. Volveremos enseguida sobre ello. En todo caso, resulta obvia la profusión simbólica que modeliza el espacio cultural de acuerdo con la lógica fantasmática de la mercancía y también del Estado. Por ello, me interesa destacar un fenómeno de gran presencia en el discurso cultural contemporáneo, muy revelador del peso simbólico de estos procesos y de la artificiosidad de la presunción de estabilidad de los lugares. Me refiero al énfasis recurrente en muchas obras de ficción contemporáneas sobre esos lugares ligados a una memoria que podríamos calificar de cultural. Evidentemente, se trata de un fenómeno asociado al giro topográfico de un sector 
importante de la producción cultural reciente. Tampoco cabe duda de la facilidad para rastrear antecedentes o incluso una tradición en este sentido, que en muy buena parte habría que hacer remontar de forma más inmediata, pero no exclusiva, al romanticismo. Cabe esperar, con todo, ciertos rasgos peculiares, especialmente en cuanto esta tendencia revela, acaso, el reverso de la pulsión conmemorativa y monumental tan propia de los poslugares más institucionalizados.

Uno de los rasgos frecuentes en esta línea de ficción contemporánea, que desde luego no minimiza el peso de la herencia indicada, es la consideración de los lugares desde un tratamiento no ajeno al motivo de la damnatio memoriae, o, más bien, de la restauración trabajosa y precaria de una memoria condenada o preterida, y que, a su vez, puede resultar altamente perturbadora. Las novelas de W.G. Sebald son un caso ilustrativo; por ejemplo, Los anillos de Saturno (Die Ringe des Saturn), que lleva el subtítulo de Una peregrinación inglesa, y muestra un recorrido evocativo y digresivo por una serie de lugares de Norfolk y Suffolk capaces de suscitar el trabajo de la memoria. El impulso para ese recorrido lo toma el narrador de su curiosidad por la figura y la obra de Thomas Browne, el autor de Hydrotaphia, or Urn Burial (1658). Es este un tratado sobre las prácticas y monumentos funerarios a través del tiempo, alentado por el hallazgo de unas antiguas urnas sepulcrales en la propiedad del señor de Crostwick Hall. El narrador de Sebald conecta su recorrido con las pesquisas sobre el paradero de los restos de Browne, casi como un eco de la pregunta que este sitúa en la dedicatoria del Urn Burial: «¿quién conoce el destino de sus huesos, o cuántas veces ha de ser enterrado?» (Browne, 2012: $167)^{4}$. Implícitamente emerge así la cuestión de la relación entre el cuerpo y otro tipo de restos o reliquias del autor y los procesos de interpretación y canonización, especialmente cuando situados en un horizonte estatal.

Por otra parte, la movilidad del sujeto en el espacio - un narrador itinerante - condiciona una configuración particular de la noción de lugar en la obra de Sebald. John Wylie, quien ha empleado la noción de espectralidad para referirse a ella, sugiere una acepción realmente sugestiva por cuanto socava algunas de las connotaciones que acompañan a la idea de lugar frente a la de espacio:

Perhaps haunting is a pre-requisite to place. That is, a place takes place through a spectral event of displacing. There is place if there is dislocation, or sudden uncertainty regarding location in space and time, uncertainty regarding even the reliability of these measurants; in other words if there is a disturbing irruption of doubt or memory, a confounding of past, present and presence all witnessed by a troubled, stricken figure, a figure haunted by this very process (Wylie, 2007: 180-181).

Se trata de una concepción que fluidifica algunas de las rigideces propias de las nociones más tradicionales del concepto de lugar, como las que remiten a las especulaciones clásicas de Yi-Fu Tuan (1997), a partir de un cuestionamiento de las conexiones demasiado lineales con las de identidad, memoria y localidad. Son reticencias o correcciones no muy alejadas de las que expresó en distintos momentos Doreen Massey (2005: 130 y ss.), aunque el lenguaje teórico sea muy distinto. Massey fue, precisamente, una investigadora muy cercana a las aproximaciones al espacio inglés de otro autor, en este caso hombre de cine, como Patrick Keiller, responsable de un celebrado ciclo de docuficciones en

\footnotetext{
${ }^{4}$ La pregunta es pertinente para tantos casos de gestión de la memoria cultural, ligándola una dimensión biopolítica del todo relevante. El caso de Rosalía Castro es elocuente en este aspecto. En relación con la poesía inglesa del siglo XIX, puede verse el estudio de Samantha Matthews (2004).
} 
torno al personaje de Robinson, un investigador solo presente a través de las alusiones en off de los narradores de estos filmes, también ellos personajes ficticios. Robinson in Space (1997), por ejemplo, muestra mediante largos planos fijos un recorrido por la Inglaterra posterior a Thatcher: un viaje de investigación en la que Robinson y su acompañante (el narrador) viajan por el país siguiendo los pasos del Defoe de A Tour through the Whole Island of Great Britain (1724-1726). Las referencias literarias son abundantes, como puede inferirse del hipotexto mencionado, pero también las alusiones a lugares literarios de distinto tipo, sujetos a esta visión que los somete a la movilidad, al entrecruzamiento de trayectorias históricas, políticas y culturales.

\section{Regreso y simulacro}

El ejercicio de memoria asociado a los lugares que incorporan Sebald o Keiller, para quedarnos en estos dos casos — ambos deudores de los caminos abiertos por el situacionismo - es de índole muy diferente a la que cabe atribuir a los poslugares literarios a que nos estamos refiriendo. Claramente, es aquel mucho más inestable, más abierto, en su formulación y articula un sujeto íntimamente disociado de lo que visita o contempla. Sin embargo, hay ciertos elementos comunes que convendría tener en cuenta también. Por supuesto, está ahí, como constituyente primordial, el papel del lugar, en su concreción y a veces en su detalle, como detonante de la evocación o de la producción de memoria. Y otro aspecto relevante es que esa relación con el lugar se construye a partir de la idea de regreso: se trata de volver al lugar en donde quizá nunca hemos estado, pero en el que ya aconteció algo que nos afecta. No es preciso, evidentemente, que el regreso sea efectivo, esto es, que haya que haber estado antes allí de una forma positiva, pero puede hablarse de retorno en tanto la visita tiene una dimensión intencional y va precedida de una expectativa y una experiencia previa que conlleva a hacer de la nueva experiencia un acto de reconocimiento, aunque sea fallido o perturbador.

Estos aspectos - la elocuencia de lo concreto y visible, junto con la idea del regreso o reviviscencia - están presentes en la constitución del poslugar, y en cierto modo se explotan, aunque tratando de asegurar y controlar su legibilidad social. La tradición teórica sobre la noción de lugar, a la que nos acabamos de referir, concibe este a partir de planteamientos de inspiración fenomenológica, donde tiende a constituir una dicotomía con el concepto de espacio. Salvando algunas vacilaciones terminológicas, el lugar sería el espacio sometido a la experiencia, y de ahí su vinculación en algunos casos, los más conservadores, con un principio estable de identidad y en otros con el de apropiación a través de las prácticas espaciales. Poco puede sorprender, por tanto, que las reflexiones contemporáneas sobre la memoria encontrasen en los lugares un apoyo determinante, tanto para sustentar en ellos la materialidad y concreción social de las prácticas colectivas de la memoria como para buscar en la dimensión memorialística una de las formas más eficaces para dar sentido a los lugares, partiendo de un entendimiento del espacio como pura materia del significante o, a veces, significante vacío.

Los análisis pioneros de Maurice Halbwachs, especialmente en La topografía legendaria de los Evangelios en Tierra Santa — publicada por vez primera en 1941—, ilustraron las posibilidades de 
esta vía de indagación cultural, y, podríamos asumirla como una exploración avant la lettre de la noción de poslugar, en un marco de carácter religioso y devocional que postula un regreso, en forma de peregrinación y de recuerdo, a los lugares de la vida de Jesús. También las consideraciones posteriores de Pierre Nora sobre los lugares de memoria pueden entenderse, al menos en parte (no todos los lugares de memoria son lugares en un sentido estricto), como una reflexión sobre la función moderna y sentido último de los poslugares en un contexto en el que la memoria tradicional se habría deteriorado hasta perder su virtualidad social y comunitaria ${ }^{5}$.

Adelaide Assmann, en su análisis de los distintos lugares como media de la memoria cultural no se detiene en esta particular situación que identificamos con los poslugares. Se aproxima mucho, sin embargo, cuando se refiere - muy significativamente - a los lugares del trauma, en especial a aquellos que se han transformado en museos o memoriales: «Underlying such places of memory that have been turned into memorials and museums is a deep-seated paradox: their conservation, which is meant to serve the purpose of authenticity, in fact leads inevitably to a loss of authenticity. By the very fact of their preservation, they have already been covered up and replaced» (A. Assmann 2011: 317). Con el término replaced, Adelaide Assmann apunta fundamentalmente a la sustitución material que transforma muchos de estos lugares, sobredeterminados por la fuerza simbólica de su localización en un aquí (aquí fue, aquí sucedió...), precisamente para conservarlos y hacerlos efectivos como instigadores de una memoria pública. Hay, sin duda, un atractivo profundo en la idea de un reemplazamiento inevitable y paradójico, tanto en el sentido de sustitución o simulación como en el de dislocación simbólica, como condición de posibilidad de los poslugares.

Tenemos un caso ilustrativo en la transformación de la antigua casa campesina de A Matanza, parte de una comunidad aldeana, en museo y sede institucional centrados en la figura de su antigua habitante, precisamente porque el énfasis retórico de sus responsables ha recaído sobre la idea de preservación. Las alteraciones materiales, ya la primera reforma de 1951, incluyeron la sustitución de la primitiva entrada principal, que comunicaba con la aldea que daba nombre y de la cual formaba parte la casa, por la actual, que convierte en fachada principal la trasera de la edificación; la erección de un cierre de piedra, que - además de enfatizar la discontinuidad del lugar - es una importante señal de prestigio constructivo en la Galicia rural; o la sustitución de las antiguas zonas agrícolas de la huerta por espacios ajardinados (Rey Lama, 1999: 22 y ss.). Por supuesto, luego vendrían otras inevitables modificaciones que, en distintas fases, afectaron al interior de la vivienda, con el objeto de adecuarla a su nueva función museística, y la adaptación o construcción de varios edificios auxiliares. Podría decirse que los cambios funcionales en la casa habían comenzado, en realidad, ya antes de la llegada de la familia Murguía-Castro, propiciados por la construcción de la vía férrea Santiago-Carril y de la estación de Padrón, inmediatas a la propiedad, que, de hecho, parece haber sido un factor decisivo para la elección de esta residencia por el matrimonio.

Cualquier lugar se halla inserto en una dinámica de transformación continua, pero buena parte de la ilusión de los poslugares consiste en trasladar una paradójica imagen de permanencia o

\footnotetext{
${ }^{5}$ Wulf Kansteiner (2002: 183) comenta de forma muy iluminadora la posición de Pierre Nora.
} 
estabilidad semántica y referencial. Para ello hay una condición constituyente: su escisión del entorno inmediato, que varía, a menudo degradándose, de acuerdo con una dinámica que es antagónica con la del poslugar. La casa de A Matanza se ha monumentalizado en la medida en que se ha aislado de la evolución paisajística y funcional de su entorno, a pesar de que su incorporación al repertorio patrimonial que el Estado decide preservar ha frustrado algunas alteraciones proyectadas ${ }^{6}$. Halbwachs (2014: 193-194) ya se había percatado de que parte intrínseca del dinamismo de la memoria colectiva es que los objetos espaciales en que esta se apoya modifiquen y, sobre todo, alteren sus relaciones con el mundo material que los rodea. Había llegado a la conclusión de que la elaboración simbólica de estos referentes espaciales los aísla como resorte para generar imágenes que subsistan por sí mismas.

\section{Formas de memoria}

Una cuestión pertinente a esta luz es la del tipo de memoria con la que se conectan los que estamos llamando poslugares literarios. Sabido es que han proliferado las distinciones entre modalidades de la memoria, más allá de la estrictamente individual, y que esas delimitaciones (memoria colectiva, histórica, pública, comunicativa, cultural, vinculante...) no siempre resultan nítidas, ni siquiera en el intento de formulación y acotación concreta de cada una de ellas. La memoria cultural indudablemente tiene mucho que ver con los poslugares, sobre todo en la medida en que atiende a las objetivaciones de la memoria que permiten estabilizar determinadas formas de recuerdo colectivo. Pero en la obra de Jan Assmann la noción de memoria cultural tiene un sentido estricto que queda desdibujado en otras formulaciones, incluso muy próximas. En este sentido más preciso, apunta a una memoria de larga duración, profundamente ritualizada, que se sostiene mediante formas de cultura estables y protocolos de comportamiento que aíslan este recuerdo del ámbito de la cotidianidad a través de una marcada orientación transcendente. «Con la memoria cultural, los abismos del tiempo se abren», llega a escribir Jan Assmann (2008: 44). De este modo, queda definida por oposición a la memoria comunicativa, asociada de manera mucho más estrecha a la vida cotidiana, a las interacciones informales y sobre todo orales en el ámbito social, con una dimensión claramente generacional y una profundidad temporal, asegura el autor, de no más de cien años. Sin embargo, Jan Assmann hubo de incluir alguna otra categoría memorialística, que conjugase, de un lado, la capacidad de objetivación y la tendencia a la formalización de la memoria cultural con un horizonte inmediato, sometido a presiones coyunturales mucho más perceptibles. Se trataría de la memoria vinculante, que contribuye a la definición de identidades colectivas con una clara dimensión normativa, como la implicada en la memoria pública del holocausto en Alemania (J. Assmann, 2008: 44 y ss.). Muchos poslugares literarios se mueven entre la memoria cultural y la vinculante: sugieren una larga duración, pero responden a formulaciones mucho más circunstanciales o, si se quiere, a procesos de índole fundamentalmente moderna en los que el Estado aparece como un actante de primer orden.

\footnotetext{
${ }^{6}$ Gonzalo Rey Lama (1999: 49), en un epígrafe titulado precisamente «A Casa Museo. O seu mundo ó redor», se refería al proyecto desestimado de hacer pasar una autopista por sus inmediaciones, pero también a las alteraciones constructivas del entorno que afectan, como se verá, al núcleo semántico del lugar rosaliano: la vistas sobre Padrón y la vega del Sar.
} 
Esta demanda de memoria asociada a lugares con una marca cultural o literaria viene, como ya se ha señalado, de muy atrás, pero se incrementa de forma notable por el impulso de las prácticas estatales o protoestatales de memorialización. Era la tesis de Heller. Podría matizarse que lo que la autora denominaba carácter cosmopolita, asociado a las prácticas de turismo cultural, no es solo un rasgo sobrevenido que se añada a estos lugares de culto laico asociados a la memoria literaria en el marco de estructuras estatales, sino que muchas veces precede a estas. El ejemplo del viaje de Lord Byron a los lugares rousseaunianos en el entorno del lago Leman en 1816 resulta ilustrativo, especialmente por cuanto se conecta con un elemento común en los viajeros vinculados a la tradición del Grand Tour, para los cuales la mediación literaria constituía un elemento esencial en su experiencia de los lugares visitados. A otra escala, habría sido también el caso del que es nuestro referente fundamental en este trabajo, la casa de A Matanza, que, previamente a su recuperación y transformación, fue incluida, aunque de manera tangencial, en itinerarios turísticos destinados a viajeros británicos. Esta cosmopolitización subyacente puede contribuir a la atención sobre la potencialidad simbólica de un determinado entorno, a veces en conjunción con el activismo cultural más circunscrito al ámbito local, que identificaba en ese mismo punto un capital cultural e identitario que exhibir ante un público foráneo. Aunque de manera larvada, eso pasó en vísperas de la Primera Guerra Mundial con la casa de A Matanza (Cabo, 2017: 983-984).

No obstante, algo diferente sucede cuando se desatan los procesos de estatalización, que implican la incorporación de estos lugares a un ámbito público, regulando a menudo su accesibilidad, cuando se promocionan a través de medios estatales y su financiación se vincula a subvenciones o se benefician de ventajas fiscales, cuando su visita se incorpora a las actividades escolares, o de otros colectivos con identidad pública reconocida, y, en suma, se asocian a redes e itinerarios que sostienen un proceso de monumentalización de la memoria de fundamento literario y cultural.

En el caso español, los referentes en este sentido son diáfanos: señaladamente, la labor de mecenazgo e intervención pública del marqués de la Vega-Inclán, en plena Restauración, con su labor entre lo privado y lo público, entre la política de recuperación patrimonial y de promoción turística, que condujo a la creación de hitos en este sentido como la Casa del Greco (1910), en Toledo, o la Casa de Cervantes (1916), en Valladolid (Menéndez Robles, 2009). Por su responsabilidad al frente de la Comisaría Regia para el Turismo y la Cultura Popular desde 1911, además de por su posición entre las elites sociales y políticas del momento, resulta patente el horizonte plenamente estatal de las iniciativas del marqués. Es obvio que nos hallamos en un contexto no muy alejado de los primeros intentos por adquirir para usos de memoria pública la casa de Rosalía. Sin entrar ahora en los detalles, la atención a la historia y antecedentes de estas iniciativas muestran, con todo, la profunda permeabilidad en estas empresas entre las iniciativas surgidas desde la sociedad civil y las prácticas institucionalizadoras del Estado, que serán decisivas a la hora de fijar un determinado modelo de lugar de memoria y dotarlo de prestigio.

En este sentido, el Estado actúa a través de procesos de cooptación, muy selectivos por su propia naturaleza, que le permiten conectar con distintas formas de memoria cultural y ligar su significado con una determinada noción de patrimonio. Podría hablarse de un proceso de apropiación, que lo es 
también de creación de sentido y de marcos identitarios más o menos precisos. Pero, al mismo tiempo, estas iniciativas, en lo que tienen de fijación de modelos, marcan también la pauta para otras iniciativas propiciadas desde la sociedad civil y orientadas, de forma más o menos inadvertida, hacia procesos de estatalización.

En este punto, importa reconocer las diferentes intencionalidades que subyacen en la constitución de los poslugares, así como la forma en que estas se modulan o transforman en el proceso que conduce a la fijación de la memoria pública. Nancy Wood ha reconocido este aspecto: «public memory whatever its unconscious vicissitudes - testifies to a will or desire on the part of some social group or disposition of power to select and organize representations of the past so that these will be embraced by individuals as their own»; a lo que añade de forma aún más precisa: «If particular representations of the past have permeated the public domain, it is because they embody an intentionality - social, political, institutional and so on — that promotes or authorizes their entry» (cfr. Kansteiner, 2002: 188). Habría que prestar atención también al tipo de intencionalidad que en cada caso «promotes or authorizes» —o, por el contrario, obstaculiza — las representaciones del pasado en el espacio público. Desde este punto de vista, los poslugares, dada la complejidad interna de su formación, pueden ser concebidos como una encrucijada de intencionalidades no siempre compatibles, aunque, en razón de su propia funcionalidad pública, tiendan a enmascarar las posibles tensiones. En este juego de intencionalidades, la cuestión de la escala resulta determinante, ya que en el establecimiento de los poslugares, especialmente cuando se produce la transición de lo íntimo o privado a lo público, hay necesariamente un proceso de escalada en la medida en que el lugar se propone como referente de experiencia a grupos cada vez más extensos.

\section{La casa de A Matanza como poslugar}

La casa de A Matanza proporciona una instancia muy ilustrativa de ello. Tras la muerte de Rosalía y el rechazo enérgico de Manuel Murguía, su marido y legatario, a que la casa fuese adquirida por instituciones públicas, a pesar de la creciente devoción por el lugar, pasó esta por años de abandono, que, al parecer, afectó incluso a parte de la biblioteca y papeles de la escritora (MiguélezCarballeira, 2014: 223-224). Murguía llegó a escribir públicamente, refiriéndose a su esposa y a la finca de A Matanza: «Nada tenía esta que ver con la casa». El airado despego del viudo de Rosalía algo tuvo que ver, posiblemente, con la órbita cultural e ideológica en que se movía el propietario de la finca, que fue pionero en marcarla con una lápida como lugar del fallecimiento de la poeta ${ }^{7} \mathrm{Al}$

\footnotetext{
${ }^{7}$ Al poco del fallecimiento de Rosalía, su familia se trasladó a A Coruña. En julio de 1900, el propietario de la casa de A Matanza, el Archivero Bibliotecario de la Universidad de Santiago de Compostela y académico correspondiente de la Real Academia Española Agustín de la Paz Bueso y Pineda, propició la colocación de una lápida de mármol, haciendo saber que allí había vivido y muerto Rosalía Castro, con el apoyo de la RAE, que publicó con tal motivo un folleto con cinco poemas de la autora. Bueso llegó a realizar gestiones ese mismo año para que los reyes se acercasen a A Matanza con motivo de una visita prevista a Santiago. Así se recoge en el diario pontevedrés El Áncora el 24 de agosto de 1900. En 1918 las diputaciones gallegas se plantearon su adquisición, con la oposición tajante y pública de Manuel Murguía, quien acusó a Bueso Pineda en una carta publicada en La Voz de Galicia el 16 de mayo de 2018 - y reproducida, entre otros lugares, en El Diario de Pontevedra el 17 de mayo de 1918 - de haber doblado el alquiler de la casa tras la muerte de la poeta y de orquestar en torno a ella una operación mercantil. Hay un pasaje significativo en la carta abierta del viudo de
} 
margen de posibles desencuentros personales, las explicaciones de Murguía sugerían una clara voluntad de control interpretativo, haciendo valer su condición de viudo de la autora; el conflicto entre encuadramientos canónicos e identitarios en pugna, con sus correspondientes apoyos institucionales; y, en tercer lugar, el nulo interés de Murguía por que la figura Castro quedase vinculada a A Matanza, y no, en cambio, a un lugar mucho más prestigioso social e históricamente como el vecino pazo de Arretén. Volveremos sobre ello.

No obstante este posicionamiento murguiano, unos particulares de filiación galleguista — José Mosquera Pérez y José Villar Granjel - acabarían haciéndose con la finca ya después de la Guerra Civil, con el objeto de constituir en ella el Patronato Rosalía de Castro, en 1947, el cual acometería la primera restauración del edificio, en 1951, que daría lugar en 1972 a la principal reforma para convertirla en casa-museo dedicada a la escritora, haciendo así de la finca de A Matanza el lugar principal de la memoria oficial del rosalianismo. Muy poco después, en 1974, fue declarada Monumento Nacional — en un Consejo de Ministros presidido, en ausencia de Franco, por el entonces Príncipe Juan Carlos - y, en 1993, Bien de Interés Cultural por la Xunta de Galicia, con consecuencias importantes para su delimitación y transmisión material, así como para su encuadramiento explícito en un marco territorial y simbólico nítidamente estatal. Luego, tras vicisitudes y obras de distinta índole, se ha producido, sobre todo en los últimos años, una ampliación notable de las actividades en torno a la casa y a la fundación que en ella tiene su sede, de la mano de su progresiva integración en una red de importante sustento público y vocación transversal en lo social e ideológico.

La propia trayectoria e institucionalización del antiguo lugar rosaliano sugiere la implicación de lo que Wulf Kansteiner (2003: 189) identifica como «mnemonic communities» con amplitudes muy diferentes — por ejemplo, la que afectó desde un primer momento a representantes de la diáspora gallega en América- , y sobre todo la activación progresiva de un aumento de su escala ligado a una búsqueda decidida de la transversalidad o consensualidad, a menudo problemática, a que antes nos referíamos. Podría decirse que se trata de una ampliación de la dimensión colectiva de una cierta memoria, más allá de los límites de las comunidades mnemónicas iniciales (en nuestro caso, círculos galleguistas conservadores de Galicia y América). Las consecuencias de este proceso han sido descritas con precisión por Kansteiner (2003: 189): «memories are at their most collective when they transcend the time and space of the events' original occurrence. As such, they take on a powerful life of their own, 'unencumbered' by actual individual memory, and become the basis of all collective remembering as disembodied, omnipresent, low-intensity memory» ${ }^{8}$. Los tres adjetivos que

\footnotetext{
Rosalía Castro en que se refiere al «caso fortuito de haber fallecido dentro de sus muros Rosalía Castro», añadiendo: «Nada tenía esta que ver con la casa en cuestión. Si había vivido en ella había satisfecho su arriendo, y, lo que es más, tenía meses antes de su muerte alquilada casa en Santiago para trasladarse a esa ciudad». Posteriormente, por ejemplo en 1928, hubo otros intentos de venta, con llamamientos a su compra para convertirla en una institución pública (Faro de Vigo, 19 de julio de 1928).

${ }^{8}$ Cursivas añadidas. Podría conectarse esta observación de Kansteiner con la siguiente apreciación de Gonzalo Rey Lama, quien fue miembro de la Fundación Rosalía de Castro y conservador de la Casa-Museo, además de presidente de la Asociación Española de Casas Museo y Fundaciones de Escritores. Tras glosar el papel del rosalianismo de posguerra como eje del galleguismo del interior durante el franquismo, sugiere: «A progresiva apertura política a partir dos 70 baleirou o Patronato [Rosalía de Castro] de tódalas connotacións políticas e, sobre todo, desa condición de refuxio do galeguismo» (Rey Lama, 1999: 19).
} 
acompañan al término memoria en la frase final son especialmente significativos. Definen en buena medida la condición de «casa de todos» que se ha atribuido a la finca de A Matanza (MiguélezCarballeira, 2014: 230-233), como expresión más rotunda de la dimensión consensual y doxística de un proceso de memorialización fundado en una adhesión emocional generalizada, pero, en efecto, de baja intensidad. Desde otro punto de vista, es un fenómeno ligado al proceso de estatalización, y de conversión en marca, que define a muchos poslugares.

No puede dejar de notarse, en este sentido, que los primeros intentos oficiales conocidos por hacerse con la propiedad datan de 1918, esto es, justo luego de un período muy concreto —entre 1891 y 1917- de continuos reconocimientos públicos y de asentamiento colectivo de Rosalía de Castro como epónimo, a partir de la deslocalización de sus restos, tras el traslado solemne de su cadáver desde Iria, en las inmediaciones de la casa de A Matanza, al convento de San Domingos en Santiago de Compostela. El adjetivo «disembodied» adquiere así un sentido muy preciso. Es el período que Francisco Rodríguez (2011: 551 y ss.) ha presentado como «etapa de asimilación» de la figura de la autora. Lo que, expresado de otra manera, se podría entender como su conversión en lo que Dovič y Helgasson han caracterizado como «cultural saint», pensando en la figura de los poetas nacionales en las distintas literaturas europeas. Es inevitable, en ese aspecto, apuntar al ejemplo, casi de manual, que proporciona un poslugar literario como la casa de A Matanza a la hora de entender cómo esta clase tan peculiar de espacios contribuye a la configuración de estos santos culturales como referentes canónicos. Téngase presente que esta clase de edificios actúan, en el marco analítico de los procesos de canonización cultural que plantean estos autores, en varias de sus dimensiones de forma simultánea: lo hace al menos como parte de la vita — en la medida en que se asocia a episodios biográficos — y del cultus - en forma de contenedor de reliquias, de monumento, de conversión en sede institucional, de escenario ritual- En este sentido, el modelo de la canonización cultural de los poetas nacionales proporciona un marco comparativo sugerente en el que incardinar un posible análisis, aunque no sea este nuestro propósito ahora

Estos y otros procesos marcan la distancia entre el lugar y el poslugar, que se construye desde la referencia identificada con el aquí del primero. Esa es una marca distintiva de los poslugares en el sentido que nos interesa: su presentación teatralizada como lugares en un sentido fuerte, esto es, como escenarios de experiencias. Como tal marca, debe ponerse al lado de otras. Así el cambio de uso del ámbito original, que tiene que ver con la teatralización, pero también con el proceso de institucionalización en sí mismo y su asociación a una práctica canonizadora de acuerdo con un determinado cultus. Todo ello implica, de cierto, lo que se podría denominar su sometimiento a un orden o a un control nuevos, lo que es tanto como apuntar a la articulación de ejes normativos de interpretación que regulan su función dentro de un marco colectivo de memoria. Otro elemento importante es que estos espacios tienden a integrarse en contextos que trascienden su localidad más estricta: sucede de manera obvia cuando se incorporan a rutas concebidas como productos preelaborados, se articulan como destinos de un turismo más o menos literario o cultural, accediendo así a formas de cosmopolitización que, a veces también, se constituye en forma de redes institucionales de fundaciones o museos literarios. Por último, señalemos otro rasgo determinante, que contribuye a 
hacer de estos poslugares verdaderos enclaves en el sentido estricto del término: su aislamiento o disociación del entorno más inmediato, precisamente por lo que tienen de representación de un espacio-otro, con una referencialidad implícita orientada en una dirección que omite su contexto próximo.

Escribe en esta línea Paola Colaiacomo (en Hendrix, 2008: 127): «For a writer's house to be transformed into a museum, it will have to undergo a preliminary process of assessment, at the end of which it will find itself severed from the housing continuity of the city, or town, or countryside, where it happens to be located. This severance is preliminary to the theatralization of the act of writing to which the museum is to be the setting». Ya hemos comentado cómo las primeras intervenciones sobre la casa de Rosalía incidieron en esta escisión del entorno. Lo más significativo con la casa de A Matanza, a diferencia de otros poslugares literarios, es que se trata sobre todo del escenario de un acto de escritura, aunque no sea este el énfasis fundamental de la disposición museística de este poslugar tal y como puede visitarse hoy en día. Es importante además conectar el acto (o actos) de escritura asociados a este lugar con el hecho nada irrelevante de que lo que hoy en día suele entenderse como la casa - casi el hogar — de Rosalía fuese en realidad una vivienda de alquiler en que la autora, ya enferma y muy desencantada, vivió con sus hijos apenas tres años, entre 1882 y el momento de su muerte en julio de 1885. Sabemos ya, por otra parte, que Manuel Murguía trató de restar importancia al vínculo de su esposa con este lugar. Por supuesto, A Matanza se situaba en un entorno de intensa emoción para la escritora, muy próximo al pazo de su familia materna y ligado a memorias muy arraigadas, pero, como tal, la finca en sí misma puede decirse que tiene un estatuto problemático como lugar. Piénsese, por otra parte, que Rosalía no tuvo jamás una residencia estable que pueda considerarse propia. Fue una autora en buena medida itinerante, que nunca hizo de sus moradas una representación directa en su obra, aunque en casos repetidos sí constituyesen el foco o el ángulo de visión desde el que conformar, hacia fuera, otros espacios representados.

No sorprende, pues, que la casa de A Matanza se sitúe en el polo opuesto de las casas de escritores conformadas por ellos mismos de manera autoconsciente como «product of a well-meditated selffashioning strategy» (Hendrix, 2008: 4) ${ }^{9}$. En estos casos, como advierte el propio Hendrix (2008: 5), desde su misma conformación se vinculan a un proceso de estabilización semántico-funcional y evocan e incluso representan la transición de lo privado a lo público. No ocurre así en la residencia última de Rosalía, dada la distancia emocional de la autora hacia ella (por no insistir en el despego de Murguía): es una casa carente de cualquier intención originaria de autoexpresión monumental. En su propia configuración y diseño material y (semi)institucional, las casas del primer tipo se proponen como poslugares y podría decirse que hay una continuidad fluida en este aspecto. En el caso de A Matanza, la situación es, en principio, distinta: el tránsito hacia el poslugar depende del aura de haber albergado a Rosalía en sus años finales. Sin embargo — el matiz es importante_- esta aura fue muy efectiva ya en vida de la autora de Cantares gallegos. Y además, aunque pueda parecer contradictorio con algunas

\footnotetext{
${ }^{9}$ Un ejemplo evidente, casi hiperbólico, de estas casas serían los edificios de la Fundación Camilo José Cela, situada a unos cientos de metros de A Matanza, también en la parroquia de Iria. Las relaciones e interferencias espaciales y de todo tipo entre ambos poslugares, así como sus respectivas relaciones con el entorno, son de sumo interés para una reflexión de esta índole.
} 
afirmaciones previas, dejó una huella crucial en la propia escritura de la autora. La escritura o, si se prefiere, la representación implica una forma de abstracción y desmaterialización que puede activar, o acompañar, el proceso hacia la constitución de poslugares, incluso en situaciones en que dichas representaciones no sean fácilmente conciliables con los marcos interpretativos asociados a los poslugares una vez constituidos.

\section{«O niño do ruiseñol»: primeros pasos del discurso público sobre A Matanza.}

Como es sabido, la obra conectada con el período transcurrido en A Matanza es, fundamentalmente, el último libro de Rosalía, En las orillas del Sar, publicado en el Establecimiento Tipográfico de Ricardo Fe, en Madrid, en mayo de 1884. Se trata de un volumen que entraña problemas filológicos todavía no resueltos y que se ha visto perjudicado por una tradición editorial deficiente. Por ejemplo, a pesar de la llamada de atención de Claude Henri Poullain (1974: 20 y ss.), los editores modernos han desdeñado por lo general la disposición editorial de los poemas de la primera edición del libro, donde estaban agrupados en secuencias y con soluciones de continuidad muy visibles (cenefas), al tiempo que han impuesto — con excepciones como la de Mauro Armiño (Castro, 1985) numeraciones ajenas al original. A ello se añade el privilegio concedido por algunos editores a la segunda edición de la obra, de carácter póstumo, impulsada por Manuel Murguía en 1909, que incorpora nuevos poemas, introduce correcciones y hace menos evidentes las agrupaciones de la primera edición. Por otra parte, el libro incluye composiciones escritas en distintos momentos, aunque está fuera de toda duda que un número decisivo se data en los años finales de residencia en la casa de la parroquia de Iria. De hecho, al menos veintidós poemas se publicaron en el diario de Buenos Aires La Nación Española entre 1882 y 1884 - escritos al parecer por encargo del propietario gallego del periódico, según su propio testimonio ${ }^{10}$ — y nueve más, los que conforman la segunda secuencia en la edición de 1884, en La Ilustración Cantábrica el primero de mayo de 1882. En cualquier caso, la propia filiación topográfica del título del poemario, así como un buen número de sus poemas —entre ellos parte de los publicados en los medios recién señalados - llevan muy perceptiblemente la marca del lugar.

Esa marca fue enseguida reconocida y tematizada en los círculos regionalistas más empáticos con Rosalía, con un impulso decisivo desde Cuba y Argentina. Algunos ejemplos son suficientes, también como muestra de la capacidad de A Matanza para objetivar y hasta podría decirse que catalizar la imagen de la autora. Incluso antes de la publicación de En las orillas del Sar, hacia octubre de 1883, un periódico santiagués de nombre El Tricornio alertaba con tintes muy dramáticos de la situación de la autora de Follas novas, a la que pintaba como enferma y moralmente abatida, «apartada en un rincón, gimiendo en el lecho del dolor, terminando su existencia [...] oscura, solitaria y en el más horroroso de los abandonos»; y solicitaba a sus lectores:

\footnotetext{
${ }^{10}$ Cuenta Barros (1885: 2) que encargó a Rosalía la escritura de los poemas que se publicaron en su periódico y que esta, más adelante, reconocería implícitamente este hecho en la dedicatoria del volumen de En las orillas del Sar que le regaló: "Nadie más que Ud. tiene derecho a la dedicatoria de este libro. Sin La Nación Española tal vez no se hubiese escrito. Reciba Ud. pues el presente ejemplar como una prueba del sincero agradecimiento de su affma.".
} 
Cuando paséis por Iria Flavia, al dirigir la vista hacia una pobre casa, oculta entre el ramaje de los árboles, descubríos porque allí se encuentra la mayor gloria del renacimiento literario de Galicia, allí se encuentra la incomparable cantora de nuestras alegrías y tristezas, sola como la estatua del dolor.

El llamamiento, en el que la imagen de la casa apartada y humilde era central, tuvo un amplio eco en otros periódicos, como, por ejemplo, el ferrolano El Correo Gallego (26 de octubre de 1883). Pronto resonó la noticia en el otro lado del Atlántico: en La Habana, el 9 de diciembre del mismo año, Waldo Álvarez Insua abría El Eco de Galicia, aludiendo al artículo de El Tricornio e instando a una suscripción entre los gallegos de Cuba para aliviar la situación de la poeta en su «modesta casa» ${ }^{11}$.

Posteriormente, Manuel Barros, el ya mencionado propietario de La Nación Española, insistió en las asociaciones de En las orillas del Sar con el entorno concreto de A Matanza en un breve artículo en el que contaba su propia visita a la poeta en ese lugar al poco de la publicación del poemario (Bouza Brey, 1961). La casa de Iria era ya una referencia. Contaba Barros como Rosalía lo recibió, amable e irónica, y, según hemos visto, le entregó un ejemplar de En las orillas del Sar con una dedicatoria en la que le reconocía su papel de estímulo para la escritura de los poemas. Pero interesa de manera especial la forma en que presentaba la casa y las asociaciones emotivas que incorporaba. Hablaba de «una casita de ruinoso aspecto rodeada de árboles y de flores», de «el tranquilo retiro de la Matanza, desde cuyas ventanas y a través de la fronda del jardín puede contemplar a su gusto este bellísimo valle de Padrón», de «una desmantelada casa perdida en un rincón de su amado valle» ${ }^{12}$. Por su parte, el periodista y farmacéutico Lisardo Barreiro en mayo de 1885 publicaría igualmente, para poner otro caso, la evocación de una visita a la escritora, ya postrada en el lecho, asociando a la casa — que califica como «templo da poesía» (1885: 3) - la escritura de En las orillas del Sar.

Podría decirse que el proceso — de alcance transatlántico — de fetichización de A Matanza como morada rosaliana, con connotaciones bastante perceptibles de índole biosocial, comienza muy pronto, durante los mismos días en que la autora de En las orillas del Sar vivía, o moría, entre sus muros. Y lo hace también desde la perspectiva de su representación iconográfica, cuando Rosalía todavía habitaba el lugar. Con la denominación de «O niño d'o ruiseñol» se reprodujo el año 1884 un grabado de la casa en el semanario compostelano El Ciclón. El grabado formaba parte de una serie - titulada «Álbum de Galicia»— que buscaba, en línea con iniciativas previas a otra escala, reproducir las «glorias monumentales, históricas y pintorescas» del país. Entre ellas figuraba la casa de la poeta, o, de otro modo, el nido del ruiseñor. Se acompañaba la ilustración, para más énfasis, de un conjunto de versos escritos por un buen número de conocidos poetas e intelectuales gallegos en homenaje a la escritora, que glosaban la imagen del nido del ave canora ${ }^{13}$.

\footnotetext{
${ }^{11} \mathrm{Al}$ parecer, la iniciativa tuvo gran éxito y se remitió a Rosalía Castro una cantidad considerable, según publicaba El Eco de Galicia el 17 de febrero de 1884. En la nota que da cuenta de la entrega del dinero en A Matanza, con la presencia del alcalde de Padrón, se da un dato precioso: «está constantemente trabajando y en la actualidad escribiendo una obra cuyos materiales remite a su esposo a Madrid, donde se está imprimiendo». Consta que la autora estaba empeñada en la composición del libro, ya instalada en Padrón, al menos desde los últimos meses de 1882 (Rodríguez, 2011: 446).

12 Repárese en la oposición «desmantelada casa» / «amado valle». Es la tensión que, como veremos, se había introducido en En las orillas del Sar, donde figuraba ya el adjetivo desmantelada para referirse a la morada de la poeta. La presencia implícita de los versos rosalianos en el texto de Barros es muy perceptible.

${ }^{13}$ El autor de las ilustraciones era José Peña, que se acompañaban — según rezaba el subtítulo de la sección— por «Descripciones hechas por distinguidos literatos de la región». Lamentablemente, no he podido localizar el número en que
} 


\section{De Murguía a Rosalía: silencio, deixis e interpretación}

La imagen que transmitirá su esposo no es ajena del todo a las que hemos visto hasta ahora, si bien su estrategia discursiva será radicalmente distinta. Manuel Murguía anticipó en la prensa a modo de homenaje fúnebre a su mujer el capítulo que incluiría poco más tarde en Los precursores. Lo hizo en las páginas de La Voz de Galicia el 17 de julio de 1885. Es un texto del máximo interés, en donde En las orillas del Sar y sus circunstancias se convierten en foco innegable de su deixis. En él, traza un perfil de Rosalía, en el que se la presenta como todavía viva, aunque muy enferma, y residente en la zona de Iria. Estamos ante una semblanza con una deixis, en efecto, muy marcada, tanto en lo temporal como en lo espacial. La perspectiva es la del presente de su composición, con una Rosalía ya tocada por «el mal que la consume y aniquila» (Murguía, 1885: 200), y el aquí de Iria, que comparte el propio Murguía: «Parece que aquí, bajo estos mismos cielos en donde, como en otro tiempo, buscó ahora su dulce refugio» (Murguía, 1885: 175) ${ }^{14}$. Ya en las líneas finales del texto, la escritura de los poemas de En las orillas del Sar se vincula directamente a esta circunstancia y este escenario: «Estas poesías escritas al pie del río que baña los campos en que pasó su infancia y en los cuales se reflejan como en las ondas los paisajes que bordan sus orillas, están llenas de las antiguas amarguras que parecen presagiar los dolores que hoy la afligen» (Murguía, 1885: 200). El poemario final de la autora de Follas novas tiene, pues, una filiación con un entorno sobredeterminado interpretativamente en lo biográfico y lo emocional desde un punto de vista discursivo, que acompaña la primera recepción de En las orillas del Sar.

En buena parte, esta sobredeterminación deriva del hecho de que la adscripción al lugar se fundamenta en la ley del retorno: un regreso, bajo el signo del refugio y el retiro, a los paisajes familiares. Sin embargo, el énfasis sobre la morada rosaliana en A Matanza que hemos visto en otros casos aparece radicalmente desplazado por Murguía. El protagonismo, que constituye todo un marco interpretativo e ideológico para la semblanza, se reserva en su caso para el pazo de Arretén, lugar de Iria, vecino del de A Matanza, que era el solar de la hidalga familia materna de la autora. En la presentación que se hace del pazo, aparece este a modo de un palimpsesto en que se sobrelee la antigua fortaleza medieval ${ }^{15}$, «la casa solariega de la décima sexta centuria», el posterior «palacio campesino del siglo XVIII» (Murguía, 1885: 172) y las «ruinas»y «silencio apenas turbado por el chillido de los pájaros» (Murguía, 1885: 173) del presente. Toda una secuencia de decadencia, presentada como la expresión de una hidalguía dimisionaria de su relación con la tierra y con el lugar. Con este trasfondo no poco dramático, Rosalía Castro es erigida, en cierto modo, en redentora, que rescata a su linaje del olvido en que había caído, y restauradora de un cierto idilio interestamental, sin omitir connotaciones

se contiene el grabado de la casa de A Matanza. Tomo la referencia del artículo "O niño d'o ruiseñol", firmado por Amador Marán (Ramón Armada Teixeiro) en El Eco de Galicia de La Habana el 15 de junio de 1884, que reproduce los versos sobre Rosalía aparecidos en El Ciclón, de autores como Eduardo Pondal, Alfredo Brañas, Juan Barcia Caballero, Gumersindo Laverde Ruiz, Eugenio Labarta o Luis Rodríguez Seoane. En el correspondiente al 5 de julio de 1884 puede verse, por ejemplo, la entrega dedicada al pórtico de San Andrés de A Coruña. En el número de El Ciclón del 18 de julio de 1884, donde se recoge la muerte de Rosalía Castro, se copian de nuevo los poemas. Se anuncia también la inclusión de un retrato de la autora que no aparece. La omisión se aclara en una nota editorial, acusando recepción de una carta de Alejandra Murguía Castro en la que pedía se prescindiese del retrato por haber sido esta la voluntad de su madre.

${ }^{14}$ Cursivas añadidas.

${ }^{15}$ Se refiere con toda probabilidad a la antigua fortaleza medieval de A Rocha Blanca, situada en el entorno de Arretén. 
cristológicas muy perceptibles ${ }^{16}$. María do Cebreiro Rábade (2012) ha mostrado el funcionamiento tropológico de la imagen de la casa en ruinas en el texto de Murguía, ligada a la idea de una restitución ideológica y cultural en un sentido identitario. La intensidad del tropo se refuerza, además, por su encarnación podría decirse que biopolítica en la persona rosaliana; de la misma manera que el contexto potencialmente representable en clave de idilio del regreso a «su dulce refugio» se ve turbado, como hemos visto, por el eco de amarguras pasadas y dolores presentes.

Tanto unas como otros tienen una dimensión biológica, pero también social, cultural e ideológica. Rábade señalaba oportunamente la fertilidad del tropo de la casa y sus representaciones en el contexto discursivo decimonónico y, de modo más particular, en el de la obra de Rosalía Castro. Por ello resulta tan llamativa la circunstancia de que el texto de Murguía, con toda su autoridad interpretativa y tan centrado en el aquí y el ahora, haga de la casa de A Matanza un punto ciego de la representación ${ }^{17}$. Se hace presente solo de manera tangencial e implícita en algún pasaje, casi como huella de su preterición. Refiriéndose a los contratiempos que nublaron su regreso, escribe, por ejemplo, Murguía: «¡Cómo la fueron a buscar al silencio de su casa y al apartamiento de su voluntario destierro!» (Murguía, 1885: 177). Esa casa adjetivada como suya - pero privada de cualquier identificación toponímica - no es la de Arretén, sino la mucho más modesta de A Matanza, esta carente de la conexión con el linaje y plenamente compatible con la noción de destierro. La estrategia discursiva llama tanto más la atención cuanto contrasta con la fetichización en marcha de la casa en otras fuentes. Pero nótese que A Matanza es, a pesar de todo, el foco subjetivo desde el que se escenifica la relación con el lugar de la autora a través de otro tropo casi tan relevante como el de la casa. Me refiero al de la ventana que abre la casa subjetivamente al exterior.: «los montes que ve desde su ventana son los mismos que veía en otros tiempos...» (Murguía, 1885: 200).

Es cierto, sin embargo, que en el prólogo que Manuel Murguía escribió para la segunda edición de En las orillas del Sar (1909) la adscripción de la obra al lugar y, sobre todo, su relación con la casa se hace mucho más explícita, aunque tampoco ahora se nombre, frente a lo que ocurre de nuevo con la mucho más prestigiosa casa de Arretén. Otra vez la imagen de la ventana resulta crucial: «Desde las ventanas de su casa veía Rosalía el atrio y los olivos que lo sombreaban, y dirigía diariamente hacia aquellas soledades sus recuerdos y sus oraciones, bien ajena, por cierto, de que pronto hallaría allí su sepultura» (en Castro, 1909: xxii). Y también la presentación de esa casa suya sin localización concreta con el origen y término de una serie de desplazamientos por el entorno definido por la emoción. Pero la casa, desde la perspectiva de Murguía, es también la expresión más radical de la intimidad, la modestia y el recogimiento de su esposa, esto es, de su no-biografía o, si se quiere, de la dimensión intrínsecamente privada que corresponde a la mujer y que, por eso, precisa de mediación ${ }^{18}$. Esta es una idea que el hombre público e intérprete de su esposa reitera en los dos textos que estamos

\footnotetext{
16 «Pero esto no fue sin tomar para sí también todas las desgracias propias de las familias que perecen» (Murguía, 1885: 174)

${ }^{17}$ No se olvide la valoración posterior de Murguía sobre la casa de A Matanza: «Nada tiene [Rosalía] que ver con la casa en cuestión». Véase la nota 6.

${ }^{18}$ Pueden leerse a esta luz formulaciones de Murguía como la que sigue: «muchas veces le dije que nadie en este mundo haría justicia a su obra, sino yo» (en Castro, 1909: viii).
} 
comentando $^{19}$. No obstante, lo más significativo en este momento es que tal estrategia de representación silenciada de la casa, como foco y referencia subyacente de un entorno localizado que, como dijimos, está definido por una sobredeterminación interpretativa, se despliega en un momento en que la casa comienza a ser un objeto de circulación pública. Dicho de otra manera, la imposición mediatizadora cobra sentido, y quizá adquiere su condición de posibilidad, cuando la casa de A Matanza esta constituyéndose como poslugar.

Pero, ¿qué sucede en En las orillas del Sar? Hasta ahora hemos considerado discursos que ponen a distancia la imagen de la poeta a través de la tercera persona, confiriéndole un aire notablemente fantasmal. Son discursos que alientan el proceso hacia el poslugar que se inicia desde la publicación del último libro de Rosalía. Si ahora nos detenemos en él, lo primero que se impone como evidencia es la gran permeabilidad, incluso léxica, entre el discurso rosaliano del lugar y el que se amplificará en las intervenciones colaterales que se suceden a partir de $1884^{20}$. Conviene señalar de entrada, no obstante, que En las orillas del Sar es un libro carente de la exuberancia topográfica y toponímica que a veces se presume y que parecería obligada en un libro donde la pulsión del lugar es tan intensa. Son muchos en el libro los poemas con una espacialidad abstracta o estilizada, donde estos referentes funcionan como correlatos objetivos o términos analógicos. Sin embargo, hay un número significativo de composiciones, que no supera la docena, en el que aflora de manera medida la designación toponímica; y algunas otras con una referencialidad reconocible, dado el contexto que estamos trazando. Entre estas se cuentan algunas cruciales en los que la casa - jamás nombrada ni identificada de forma precisa - se convierte en su núcleo deíctico y tropológico, y acaso del libro en su conjunto.

Es el caso del extenso poema inaugural de la edición original de la obra: Orillas del Sar, una de las diez únicas composiciones del volumen que llevan título. El arranque del poema nos sitúa —una vez más - ante un acto de visión hacia fuera desde un interior no representado: «A través del follaje perenne / ... / desde mis ventanas veo / el templo que quise tanto» (Castro, 1985: 93). Enseguida, ese mirar al exterior desde dentro de la casa subrayará una dimensión tropológica esencial que enfrenta el punto de observación a lo que se contempla en la distancia: «Y afligido mi espíritu, apenas / De su cárcel estrecha y sombría / Osa dejar las tinieblas / Para bañarse en las ondas / De luz que el espacio llenan» (94). Hasta tal punto se incide en esta tensión, que, frente a ese entorno hacia el que se proyecta el sujeto lírico, el lugar distanciado desde el que se mira tiene la marca de la alienación: «Cual si en suelo extranjero me hallase, / Tímida y hosca contemplo / Desde lejos los bosques y alturas» (94). Simultáneamente, esa visión, que arrastra al sujeto lírico fuera del recinto presentado como una cárcel, se vincula a una experiencia y a una emoción ligadas al pasado y a la memoria. Pero es una emocionalidad antagónica e indecisa, que se resalta a lo largo de todo el poema. Solo a partir del verso 61 la mirada se transmuta en acción para iniciar un paseo por el «camino antiguo», «la senda amiga»,

\footnotetext{
${ }^{19}$ En el que forma parte de Los Precursores se incluye un aserto muy reiterado: «La mujer deber ser sin hechos y sin biografía» (Murguía, 1885: 176). Y en el prólogo a la edición de 1909 de En las orillas del Sar: «Después de todo, la vida de una mujer, por muy ilustre que sea, es siempre bien sencilla. La de Rosalía, como la de cuantas se hallan en su caso, se limita á dos fechas : la de su nacimiento y la de su muerte; lo demás sólo importa á los suyos» (en Castro, 1909: xx).

${ }^{20}$ La dimensión fantasmática que adquiere la figura de Rosalía Castro en este contexto discursivo depende mucho del hecho de que su correspondencia, sin duda muy nutrida también en este período, fuese destruida en gran parte por Manuel Murguía (Naya, 1959: 18-19).
} 
que conduce, por primera vez, a un despliegue toponímico (Trabanca, la Torre, la Presa, Fondóns, Miranda: todos en cursiva en la edición de 1884; junto a Sar, Padrón o Iria Flavia).

La experiencia enfatizada por las evocaciones que suscita el recorrido es tanto como la disrupción del idilio, representado con fórmulas afines a la tradición bucólica. Y esa conciencia oscura provoca finalmente el regreso al punto de partida del recorrido, «A mi morada oscura, desmantelada y fría», equiparado en los versos finales al «negro nido del ave agorera», al «antro escondido» de la fiera, al «sepulcro» del muerto, al «olvido»y al «desierto» (99). ¿Cabe mayor antinomia con el tropo del nido del ruiseñor — o niño do ruiseñol— con que fue celebrada A Matanza en la prensa de la época?

En cualquier caso, las referencias a la casa, en contraste con el acto de localización expreso en el título del poema y las identificaciones toponímicas en su interior, son imprecisas, pero totalmente compatibles con las numerosas alusiones contemporáneas y con el señalamiento y la deixis que Murguía entroniza en su capítulo de Los precursores. Ese primer poema, por otra parte, establece una continuidad muy clara con otros, que reproducen el recorrido trazado en él por los alrededores de la casa, como «Del antiguo camino a lo largo» (127) o «Camino blanco, viejo camino» (146). Hay otras composiciones que, de nuevo sin precisión alguna, definen ámbitos que pueden asociarse con $\mathrm{A}$ Matanza, así «En su cárcel de espinos y rosas» (138-139), donde se reitera la imagen de la finca como prisión, que ya hemos visto, o «En mi pequeño huerto» (168); o también «Cenicientas las aguas, los desnudos» (140-141), que presenta a la voz poética otra vez percibiendo el entorno desde el interior de su vivienda: «Yo desde mi ventana...». El último poema de la colección, por otra parte, retoma el término ya utilizado en el inaugural: «En el dintel oscuro de mi pobre morada» (223).

Por supuesto, hay otras localizaciones en los poemas del volumen, pero el énfasis sobre el lugar concreto de los «lares primitivos» y la función con respecto a ellos de la morada de la poeta son muy relevantes y no menos problemáticos. Coinciden los poemas de En las orillas del Sar con la estrategia discursiva de Murguía en no nombrar la casa, aun cuando esta se parece mucho a un punto focal deíctico e ideológico. Pero frente a las valoraciones de Murguía, que, en el prólogo a la segunda edición del poemario, presentaba a su esposa «contenta en la soledad de su casa, tranquila en sus medianías, satisfecha viendo crecer sus hijos y siendo con ellos dichosa» (en Castro, 1909: xii-xiii) —esto es, sumida en el ámbito privado de la no biografía femenina-, la morada representada por Rosalía posee una muy evidente carga distópica. Es ilustrativo el que es acaso el único poema del volumen desarrollado en un espacio interior — «Cuido una planta bella» (173) - , en el que las nociones de oscuridad y angostura identifican el lugar. Podría incluso conectarse esa figuración de la casa con el sentimiento de alienación e imposible consonancia con los lares «primitivos», que, en buena medida, es el resultado de procesos culturales, sociales y económicos que escapan — no sus efectos - a la representación en la obra rosaliana (Labrador, 2015).

El retorno es, por tanto, uno de los pivotes semánticos y pragmáticos de este libro de Rosalía Castro. La idea del regreso implica en sí misma una potente dimensión espectral, por cuanto apunta a una experiencia pasada imposible de materializar a través de la vuelta al lugar. Si atendemos al siempre sugerente Jacques Derrida (1994: 11), es, de hecho, quien regresa el que acaba por adquirir la condición de espectro, envuelto en un proceso inacabable de retorno sin posibilidad de llegar nunca del todo. 
Este es seguramente el núcleo de la obra última de Rosalía. Y su máxima expresión es la casa ajena, la casa en disputa, convertida en el foco central de la mirada y en factor de disrupción de la continuidad con el entorno y quiebra del idilio. Dejando aparte a Murguía, que sobre todo obvia o disimula estas circunstancias, los discursos colaterales sobre la casa realizan un acto de radical significación cultural e ideológica, consistente en la inversión de la mirada hacia el propio edificio que subraya su condición de enclave definitivamente escindido de su entorno en su condición de poslugar.

\section{Algunas conclusiones}

Es el momento de ir llegando a algunas conclusiones, aunque sean, como suele suceder, provisionales en la medida en que dependen de matizaciones, análisis e interpretaciones más completos y precisos, así como de la continuación de algunas de las vías que se han abierto en el presente trabajo. Las principales conclusiones podría ser, al menos por el momento, estas:

1. Las relaciones entre literatura y lugar resultan ser, al menos en casos como el aquí valorado, íntimas y enormemente complejas. No se trata de reconocer la mera referencialidad interna de algunos textos, sino de dar cuenta en todo su alcance de la involucración, a menudo problemática, en procesos de conceptualización y semantización de los espacios desde una perspectiva múltiple - cultural, identitaria, ideológica, política...-. Son procesos que afectan a la articulación general de los campos literarios y a sus distintos agentes, con la consiguiente adopción de posiciones. En ellos, además, la conformación de poslugares, en el sentido que he tratado de precisar y que implica la inscripción espacial de lo literario, resultar un factor decisivo, entre otros motivos, por el gobierno complejo de las emociones y las formas de memorialización colectiva que implican.

2. En lo que se refiere a la casa de A Matanza, nos hemos acercado a ella desde el entrecruzamiento de varias dimensiones discursivas, que incluyen — de manera muy limitada, en esta ocasión - la representación desde el interior de los poemas, otras textualizaciones contemporáneas y el momento inicial de la tradición iconográfica vinculada a ese lugar. Entre ellas no cabe la concordancia, y, de hecho, el ahondar en las tensiones que sugieren es la condición de cualquier interpretación no meramente apropiadora y, en última instancia, abusiva. La figuración de este espacio en los poemas rosalianos se realiza desde la reticencia, pero haciendo de la morada un foco decisivo de la representación del entorno familiar y querido, regido por la noción de retorno, y con una carga tropológica muy poderosa. Por otro lado, la asociación con un lugar muy sobredeterminado interpretativamente, como el entorno de Arretén en Iria, se convertirá en clave, desde la óptica de Murguía, para el encuadramiento canónico de En las orillas del Sar, y a su través del conjunto de la obra de Rosalía. Por último, desde un primer momento, ya en vida de la poeta, la 
referencia icónica de A Matanza funcionó, de la mano de una comunidad mnemónica que se iría ampliando progresivamente, como icono y figura de memoria de quien muy pronto se deslizó a la categoría de santa cultural. Ello favoreció la atenuación de otras posibles lecturas — sociales, ideológicas o de género_-, que aquí hemos sugerido.

3. La consideración del caso de A Matanza abre nuevas perspectivas para el análisis y tipologización de los poslugares literarios y, especialmente, de las casas de escritores. En particular, interesa constatar que, si bien nunca fue el resultado de un proceso de self-fashioning, tampoco es meramente consecuencia de una postproducción diferida y desconectada de la realidad cultural inicial de ese espacio. Más bien, de ahí su eficacia, ilustra una instancia en la que el poslugar se vincula a procesos en los que se suceden en una continuidad conflictiva - también desde la perspectiva nacional y lingüística - formas de memoria comunicativa, vinculante y cultural, con los distintos ritmos temporales y amplitud de comunidades nemónicas que les corresponden. Son procesos que se asientan en una a menudo ignorada disociación simbólica, material y perceptiva del entorno; y también en una solución de continuidad con la emotividad rosaliana.

4. Todo lo anterior puede situarse en la perspectiva de la relación de la literatura con el Estado. Hemos visto que la fabricación del poslugar puede tener un desarrollo que se origina desde el momento de su misma experiencia como lugar, y que la propia presentación literaria incide en ello a través de la abstracción y desmaterialización ligadas a la ficción. Pero para su constitución efectiva precisa convertirse en figura de memoria. El Estado - en un sentido lato, no meramente administrativo- tiene en la memoria cultural una de las formas más eficaces de legitimación, y podría decirse que los poslugares adoptan en su conformación una pauta memorialista de orden estatal. Es lo propio de las casas de escritores como bien cultural; luego, para su continuidad institucionalizada, precisan de su inscripción, más o menos rotunda, en los aparatos estatales, a veces con la participación mediadora de la sociedad civil. Uno de los resultados, o condiciones, es la ampliación las comunidades mnemónicas a través de retóricas de consenso y prácticas doxísticas. También se implica una minoración de su carga emocional, lo que, por otra parte, las acerca a la cosmopolitización y mercantilización, a través de su reconsideración como producto turístico.

En suma, la casa de A Matanza, desde la parcialidad de un caso concreto, muestra aspectos del funcionamiento cultural e ideológico de los poslugares literarios muy atendibles. No solo sugiere la complejidad y dinamismo del concepto de lugar en relación con estos entornos, sino que hace evidente su condición de encrucijada discursiva que disimula tensiones a veces de notable calado, a pesar de la pretensión de consenso asociado a su condición de figura de una memoria pública. En este sentido, 
ilumina facetas importantes de la relación entre literatura y Estado, ligadas a la proyección comunitaria de un patrimonio literario estabilizado institucionalmente y al funcionamiento de ese Estado como horizonte de consagración pública. En una línea contraria, se abre también la consideración de la condición espectral que adquiere la ficción literaria o poética que sostiene la legitimidad pública de estos lugares. Frente a la apropiación poderosa, que encubre con más o menos éxito los conflictos simbólicos, la espectralidad de la escritura se convierte en una vía posible y eficaz de crítica cultural.

\section{Referencias bibliográficas}

Assmann, A. (2011): Cultural Memory and Western Civilization. Arts of Memory. Nueva York, Cambridge University Press.

Assmann, J. (2008): Religión y memoria cultural. Diez estudios. Ed. y trad. M. G. Burello - K. Saban. Buenos Aires, Lilmod.

BARREIRO, L. (1885): «Unha visita a Rosalía Castro», El Eco de Galicia. Revista Semanal de Ciencias, Artes y Literatura (La Habana), 153, 31 de mayo, pp. 2-3.

BouZA BREY, F. (1961): «Manuel Barros, escritor emigrado, amigo de Rosalía, y los orígenes del libro En las orillas del Sar», Cuadernos de Estudios Gallegos, 49, pp. 218-239.

Barros, M. (1885): «Páginas de un libro», El Eco de Galicia. Revista Semanal de Ciencias, Artes y Literatura (La Habana), 162, 2 de agosto, pp. 2-3.

Browne, T. (2002): La religión del médico. El enterramiento en urnas. Ed. y trad. J. Marías. Barcelona, Debolsillo, $2^{\mathrm{a}}$ ed., 2012.

Cabo AseguinolazA, F. (2017): «Guías de viaje y novelas en la construcción literaria de Compostela: las estudiantinas como ficciones autoetnográficas», Bulletin of Hispanic Studies, 94/9, pp. 971986.

_- (en prensa): «Memoria, (pos)lugar y biopoder en un thriller literario: A memoria da choiva, de Pedro Feijoo», en K. MoszCZyŃSKA-DÜrst y A. CALDERÓn PUERTA, eds., Memoria encarnada. Varsovia, Instituto de Estudios Ibericos e Iberoamericanos de la Universidad de Varsovia. DOI de la prepublicación: 10.13140/RG.2.2.27219.68641.

CASTRO, R. de (1909): Obras completas de Rosalía de Castro I. En las orillas del Sar. Prólogo de Manuel Murguía. Madrid, Librería de Pueyo.

(1985): En las orillas del Sar. Ed. M. Armiño. Barcelona, Plaza \& Janés.

DERRIDA, J. (1994): Spectres of Marx. Londres, Routledge.

Dović, M. - Helgasson, J. K. (2017): National Poets, Cultural Saints. Canonization and Commemorative Cults of Writers in Europe. Leiden, Brill.

Halbwachs, M. (2014): La topografía legendaria de los evangelios en Tierra Santa. Estudio de memoria colectiva. Trad. Ramón Ramos. Madrid, Centro de Investigaciones Sociológicas.

Heller, A. (2001): «Cultural Memory, Identity and Civil Society», Internationale Politik und Gesellschaft, 2, pp. 139-143.

HENDRIX, H., ed. (2012): Writer's Houses and the Making of Memory. Nueva York, Routledge. 
160 | Tropelías. Revista de Teoría de la Literatura y Literatura Comparada, número extraordinario 4 (2018) Fernando Cabo Aseguinolaza

KAnsteIneR, W. (2002): «Finding Meaning in Memory: A Methodological Critique of Colletive Memory Studies», History and Theory, 41, pp. 179-197.

LABRAdOR MÉndeZ, G. (2016): «Las Églogas de la Acumulación Originaria. Paisajización, desposesión y memoria demopoética en Rosalía de Castro (1868-1884)», en F. MARTínEZ y K. SORIANO, eds., Revisitar el costumbrismo: cosmopolitismo, pedagogías y modernización. Frankfurt am Maine, Peter Lang, 2016, pp. 251-269.

MASSEY, D. (2005): For Space. Londres, SAGE.

Matthews, S. (2004): Poetical Remains. Poet's Graves, Bodies, and Books in the Nineteenth Century. Oxford, Oxford University Press.

MenÉNDEZ Robles, M. L. (2009): «Mecenazgo e implantación de las Casas-museo en España», en J. I. GIL PIÑERO, ed., Museos y mecenazgo: nuevas aportaciones. Madrid, Universidad Rey Juan Carlos, pp. 51-76.

Murguía, M. (1885): Los precursores. A Coruña, Imprenta de La Voz de Galicia.

MiguÉlez-CARbAlleirA, H. (2014): «A casa de Rosalía, a Rosalía da casa: historia, discurso e representación na Casa-Museo Rosalía de Castro», en R. Álvarez, A. AngueIRA, M. do C. RÁbade y D. VILAVEDRA, eds., Rosalía de Castro no século XXI. Unha nova ollada. Santiago de Compostela, Consello da Cultura Galega, pp 219-234. DOI:10.17075/rcsxxi.2014.

NAYA, J. (1959): Inéditos de Rosalía. Santiago de Compostela, Patronato Rosalía de Castro.

Pollain, C. H. (1974): Rosalía Castro de Murguía y su obra literaria. Madrid, Editora Nacional.

RÁBADE VILlAR, M. C. (2012): «Una casa en disputa. Rosalía de Castro entre la ruina y la restitución», Revista de Estudios Hispánicos, 46/3, pp. 29-54.

Rey Lama, G. (1999): Vinte quilómetros ó sur de Santiago: A casa-museo de Rosalía. Padrón, Fundación Rosalía de Castro.

RodríGueZ, F. (2011): Rosalía de Castro, estranxeira na súa patria. A Coruña, Asociación SocioPedagóxica Galega.

TUAN, Y.-F. (1997): Space and Place: The perspective of Experience. Minneapolis, University of Minnesota Press.

Watson, N. J., ed. (2009): Literary Tourism and Nineteenth Century Culture. Londres, Palgrave Macmillan.

WYLIE, J. (2007): «The spectral geographies of W.G. Sebald», Cultural Geographies, 14, pp. 171-188. 